\title{
Analisando efeitos de diferentes contextos sintáticos e discursivos sobre as funções dos sinais de apontação em libras
}

\author{
Discussing the effects of different discursive and syntactical contexts \\ on the functions of the pointing signs in Libras
}

\begin{abstract}
Anderson Almeida-Silva ${ }^{1}$
Resumo: Neste artigo proponho uma discussão sobre os contextos discursivos e sintáticos em que há variação na leitura categorial dos sinais de apontação em libras, e os possíveis efeitos destes para alteração de propostas anteriores (ALMEIDA-SILVA, 2019; 2021) que fiz sobre a categorização das apontações em libras. Utilizando dados de diferentes tipos e fontes conseguimos identificar que: $i$. o contexto de produção dos sintagmas nominais, se 'out-of-blue' ou anafóricos, tem influência na leitura categorial dos sinais de apontação, mas que ii. a coordenação de nomes no espaço de sinalização não privilegia a leitura demonstrativa, como se hipotetizava. Por fim, a categorização proposta por Almeida-Silva (2019;2021) pode ser mantida sem prejuízos, apesar dos efeitos variacionais oriundos dos contextos supracitados.
\end{abstract}

Palavras-chave: Categorização. Sinais de apontação. Contextos sintáticos de produção. Língua Brasileira de Sinais - Libras

Abstract: In this paper I propose a discussion about discursive and syntactic contexts that allow for varying readings to the pointing signs categories in Libras, and how they could alter anterior proposals (ALMEIDA-SILVA, 2019;2021) I made for the categorization of the pointing signs in Libras. Using data from different types and sources I find that: $i$. the context of production, if out-of-the-blue or anaphorical, has an influence on the categorial reading of the pointing signs, however $i i$. coordination of nouns realized in the signing space does not privilege for the demonstrative reading, as I hypothesized. In sum, the categorization proposed by Almeida-Silva $(2019 ; 2021)$ can be maintained without alterations, despite of the varying effects raised from the above contexts.

Keywords: Categorization. Pointing signs. Syntactic Contexts of production. Brazilian Sign Language - Libras.

\section{Introdução}

Este trabalho tem por objetivo trazer à baila uma problematização sobre a questão das categorias dos sinais de apontação com o indicador em libras, também referidos aqui como IX ह.

Conforme propus anteriormente em Almeida-Silva $(2019 ; 2021)$ os sinais de apontação em libras podem ter suas categorias morfológicas identificadas a partir de dois níveis, o fonológico e o sintático como na tabela 1 a seguir.

\footnotetext{
${ }^{1}$ Universidade Federal do Delta do Parnaíba, Departamento de Ciências Biológicas, Parnaíba, PI, Brasil. Endereço eletrônico: andersonalmeida@ufpi.edu.br.
} 
Tabela 1 - Distinção fonológica e sintática das categorias dos sinais de apontação em libras

\begin{tabular}{|l|l|l|}
\hline \multicolumn{3}{|c|}{ FUNÇÕES DA APONTAÇÃO EM LIBRAS } \\
\hline \multicolumn{2}{|c|}{ DISTINÇÃO FONOLÓGICA } \\
\hline \multicolumn{2}{|c|}{ Formas não-homófonas de IX } & \multicolumn{1}{|c|}{ Formas homófonas de IX } \\
\hline $\begin{array}{l}\text { - Demonstrativos não-gramaticalizados }{ }^{2} ; \\
\text { - Advérbios de lugar (IX }\end{array}$ adv $)$ & $\begin{array}{l}\text { - Pronomes pessoais; } \\
\text { - Demonstrativos gramaticalizados (IX pós- } \\
\text { nominal) } \\
\text { - Artigos definidos (IX pré-nominal) }\end{array}$ \\
\hline
\end{tabular}

Fonte: Almeida-Silva $(2019 ; 2021)$.

Como se pôde observar na tabela 1, propus anteriormente que haveria três tipos de apontação em libras que são fonologicamente distintas, a saber, as apontações que apontam literalmente para objetos no espaço real ou no espaço mental ${ }^{3}$ como nos exemplos em (1), as apontações adverbiais locativas porque apontam num plano transversal que seria distinto do plano horizontal (2) e as apontações laterais no plano horizontal que são idênticas entre si (homófonas) (3), (4) e (5). Estas três últimas ocorrências de apontação, por serem homófonas, só teriam sua categorização morfológica desambiguizada na sintaxe. A proposta é que a IX isolada em posição argumental de um verbo é um pronome pessoal (3), a IX pós-nominal acompanhando um nome em posição argumental é um demonstrativo gramaticalizado (4) e a IX pré-nominal acompanhando um nome em posição argumental um artigo definido (5). Portanto, estas últimas seriam dependentes da ordem e da distribuição sintática para que seja identificada sua categorização ${ }^{4}$.

\section{(1) Demonstrativo não gramaticalizado}

a. Objeto real

Situação: Numa galeria de arte, há um quadro do Van Gogh:

A: $\langle\mathbf{I X}\rangle_{\text {apontando-ostensivamente-para-o-quadro BONITO }}$

'Este/esse/?o quadro é bonito'

b. Objeto mental

\footnotetext{
${ }^{2}$ Embora soe contraditório dizer que uma categoria gramatical, tal qual o demonstrativo, não é gramaticalizada. Aqui, o termo 'não-gramaticalizado' quer dizer que seu significado depende inteiramente do objeto, presente ou ausente no espaço de sinalização, para o qual se aponta, ou seja, não está previsto somente pela gramática.

${ }^{3}$ Estes conceitos de espaço mental e real ficam mais claros nos exemplos em (1a) e (1b), mas não guardam relação com o conceito de espaços mentais como concebido em Liddell (2003)

${ }^{4}$ De forma parecida ao que ocorre nos casos chamados de derivação imprópria pela gramática tradicional.
} 
Situação: Alguém sinalizando para outro sobre a posição relativa de 3 escolas numa determinada cidade.

A: $\langle\text { IX }\rangle_{\text {apontando-para-uma-das-escolas }}$ MELHOR DO-QUE $\langle\text { IX }\rangle_{\text {apontando-para-outra-das-três-escolas }}$

'Esta/Aquela/Ela é melhor do que esta/aquela/ela'

(2) Advérbio de lugar

EU MORO IX(lá) SÃO-PAULO

'Eu moro lá/ali/aqui em São Paulo'

(3) Pronome pessoal

IX SABER LIBRAS

'Ele/a sabe libras'

(4) Demonstrativo

LIBRAS-a IX-a ${ }^{\mathbf{5}}$ IMPORTANTE SURDO

'A libras (esta língua) é importante para o surdo'

(5) Artigo definido

EU QUERER-NÃO PREJUDICAR IX.PL SURDO

'Eu não quero prejudicar os/estes surdos'

No entanto, percebemos que esta classificação proposta, em alguns outros contextos sintáticos ou discursivos, poderia ser questionada à primeira vista, pelo principal fato de as mesmas apontações poderem ter leituras convergentes com outras categorias morfológicas diferentes das que propus ali. Por isso, para fins de uma descrição formal da libras, tendo como base a teoria gerativa (CHOMSKY, 1986; 1995 e outras publicações) não se conceberia uma aquisição em que as crianças surdas não reconheçam minimamente as funções desempenhadas por cada tipo de apontação, e a por isso, é relevante a investigação sobre a categorização dos sinais de IX na língua, tendo em visto os contextos de uso e aquisição.

A questão que norteia esta discussão então é: a classificação proposta na tabela 1 pode ser mantida sem perder de vista a interação com outros fenômenos linguísticos e discursivos? Tentarei trazer evidências para defender que sim e para isso, vamos explorar dois casos: o primeiro, na seção A função possesiva da apontação lateral, vai analisar como em alguns contextos, a apontação lateral pode ter uma leitura de item possessivo, e o segundo, na seção $A$ coordenação de sintagmas e a apontação, lançarei questões sobre como a coordenação de sintagmas utilizando a IX poderia ser confundido com uso demonstrativo da apontação.

\footnotetext{
${ }^{5}$ Nota sobre a transcrição e as glosas: i. os sinais são representados em letras maiúsculas; ii. os índices subscritos (-a) indicam que o sinal foi localizado em um ponto arbitrário no espaço ao redor do sinalizador; iii. o item IX indica a apontação para os espaços laterais; iv. o item IX-a indica a apontação correferente ao sinal localizado no espaço de sinalização e v. IX.SG=apontação retilínea/singular e PL= apontação em arco/plural.
} 


\section{Metodologia}

Para as análises foram utilizados dados sinalizados de quatro diferentes tipos: $i$. dados naturalísticos, ou seja, aqueles em que o sinalizador é gravado sem nenhum propósito específico; $i i$. dados espontâneos, aqueles em que o sinalizador produz um texto sinalizado sem que tenha sido solicitado a produzi-lo, como por exemplos os vídeos de sinalização da internet ${ }^{6}$, vídeos de opinião, etc; iii. dados semiespontâneos, aqueles nos quais o sinalizador conversa com outro sinalizador sobre temas previamente selecionados em uma situação um pouco mais monitorada e $i v$. os dados elicitados, aqueles em que o pesquisador faz perguntas diretas sobre a estrutura das sentenças e explora a intuição e o conhecimento metalinguístico que o falante tem sobre a língua em análise.

Os dados do tipo $i$ correspondem aos dados cedidos pelo INES $^{7}$ de gravações de conversas de surdos no pátio da escola durante o recreio, os do tipo ii são dados de 10 vídeos sinalizados selecionados de forma aleatória em fóruns sinalizados na internet e com duração de 3' a 7' cada um $^{8}$. Estes vídeos foram analisados e as ocorrências das apontações IX em sintagmas nominais (SNs) na libras foram anotadas, por minuto, para cada vídeo, destacando se o item estava em posição de sujeito $(\mathrm{S})$, objeto(O) ou uma posição não $\operatorname{argumental}(\mathrm{N})$, conforme tabela em anexo a este texto. Os dados do tipo iii são os dados do Corpus da Libras da UFSC. Os dados do corpus estão anotados no software ELAN - Eudico Linguistic Annotator $^{9}$ - pela equipe de pesquisadores da UFSC. Os dados que utilizei compreendem cerca de $2 \mathrm{~h} 30 \mathrm{~m}$ de entrevistas realizadas com surdos sobre temas como 'implante coclear e tecnologia' e 'educação de surdos'. Os dados do tipo iv são dados de uma entrevista realizada com cinco alunos surdos do curso superior em Letras Libras da UFAL. A entrevista era semiestruturada pois os pontos que seriam apresentados foram previamente estabelecidos, embora houvesse total liberdade para o participante sugerir ou alterar o desenrolar da conversa. A entrevista consistiu basicamente em levantar dados de intuição sobre a aceitabilidade das sentenças.

\footnotetext{
${ }^{6}$ Disponibilizo, em anexo, a tabulação dos dados destes vídeos, com as ocorrências de sintagmas nominais anotadas, minuto a minuto.

${ }^{7}$ Agradeço a pesquisadora Profa. Dra. Solange Rocha pela cessão desse material para minha pesquisa.

${ }^{8} \mathrm{Na}$ tabela em anexo, o leitor pode ter acesso a miniatura dos vídeos como uma referência dos seus autores. Os vídeos de números 3 a 8 constavam na página do Facebook denominada Sociedade em Libras https://www.facebook.com/ASociedadeemLibras/ e atualmente, alguns não estão mais disponíveis online. Já os vídeos de números 1, 2, 9 e 10 não possuem links fixos, foram vídeos de redes sociais que o autor deste texto solicitoupessoalmente a autorização de cada um dos surdos para a utilização específica para as suas análises.

9 O ELAN é um software, uma ferramenta profissional para anotar e transcrever de forma manual e semiautomaticamente gravações de áudio ou vídeo. Possui um modelo de análise baseado em trilhas (layers) que suporta que sejam feitas anotações de vários níveis e de vários participantes por segundo.
} 


\section{Problematizando sintaticamente as apontações em libras}

A função possessiva da apontação lateral

Trago aqui para a discussão casos nos quais as apontações que definimos como demonstrativos gramaticalizados (IX pós-nominal) e artigos (IX-pré-nominal) receberia a leitura de pronome possessivo.

Em libras, e em várias línguas de sinais (LS) (ALIBAŠIĆ \& WILBUR, 2006; CORMIER \& FENLON, 2010; ABNER, 2012) é comum que o item possessivo possua uma configuração de mão distinta das formas pronominais, que como vimos no exemplo em (3) deste texto, utiliza a apontação com o indicador . E por isso, os itens possessivos nas LS são geralmente analisados como os únicos itens que apresentariam uma marcação morfológica de caso, por conta da configuração de mão distinta do pronome pessoal. No entanto, parece que em alguns contextos, o uso das formas pronominais para veicular posse estaria em variação livre como o pronome possessivo.

Embora a libras empregue configurações de mão distintas para os seus pronomes possessivos (configuração de mão em $\mathrm{P} \mathbf{d}$ ), os sinais de IX como possessivos, como no exemplo em (6).

A partir da intuição dos surdos entrevistados, a leitura de posse ocorreria em libras mais com os pronomes pessoais de $1^{\mathrm{a}}$ (IX-1) e $2^{\mathrm{a}}$ (IX-2) pessoa, como em (6) e (7) respectivamente, contudo, como possível resultado da mudança sofrida pelo pronome de $3^{\mathrm{a}}$ pessoa para os usos como artigo e demonstrativos gramaticalizados em libras, a leitura de posse nem sempre é clara com a apontação de $3^{\mathrm{a}}$ pessoa (IX-3), como pode ser evidenciado pelo contraste em (8a-b), pois nos contextos 'out-of-the-blue ${ }^{10}(8 \mathrm{a})$ a interpretação preferencial de IX-3 pré-nominal seria a de um artigo definido, no entanto, em um contexto anafórico (8b), a função pronominal é mais saliente e seria beneficiada, por isso, a interpretação preferencial neste caso seria a possessiva.

\footnotetext{
${ }^{10} \mathrm{O}$ termo em inglês designa contextos inéditos, 'do nada' e que ocorram sem nenhuma informação tenha sido disponibilizada anteriormente.
} 
(6) Pronome $1^{a}$ pessoa

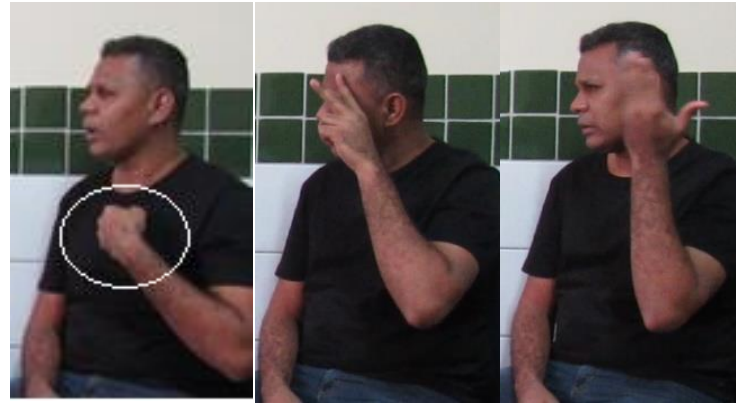

[IX-1 HISTÓRIA PASSADO]

Literalmente: 'Eu história passado.'

'Minha história do passado'

(7) Pronome $2^{a}$ pessoa

[IX-1] ESQUECER [IX-2 NOME] DESCULPAR

Literalmente: 'Eu esqueci você nome, desculpa.'

'Eu esqueci seu/teu nome, desculpa!'

(8) Pronome $3^{a}$ pessoa

a. Contexto 'out-of-the-blue'

\section{[IX-3 VOVO] SABER LIBRAS}

Literalmente: 'Ela vovó sabe libras'.

a. 'A vovó sabe libras'

b. ?'A vó dela sabe libras'

(dados da entrevista)

\section{b. Contexto anafórico}

\section{A: IX-1 AMIGO 3-FALAR-1 AMANHÃ FESTA FAMÍLIA DELE}

'Meu amigo me falou que amanhã tem uma festa com a família (dele)'

\section{B: [IX-3 FAMÍLIA] SABER LIBRAS?}

a. 'A família dele saber libras?'

b. 'A família sabe libras?'

\section{A: NÃO-SABER CERTO}

'Não tenho certeza!'

(dados da entrevista)

A mesma interpretação ambígua entre possessivo e artigo definido também ocorre com IX pós-nominal. Note que nos contextos de retomada a seguir, tanto o IX pré-nominal em (9), como o IX pós-nominal (10) tem leitura preferencial de posse se retomarem algum referente 
mencionado. Em (10) a leitura anafórica é licenciada por bridging ${ }^{11}$, assumindo que qualquer criança deve estar contida numa família. O fato de o elemento ter a leitura possessiva em alguns contextos não invalidaria, então, nossa análise de que IX pré-nominal seja de fato um artigo definido e IX pós-nominal um demonstrativo gramaticalizado, no entanto, reconhece-se que o contexto discursivo de produção pode ter uma interferência na preferência da interpretação da categoria do item.

(9)

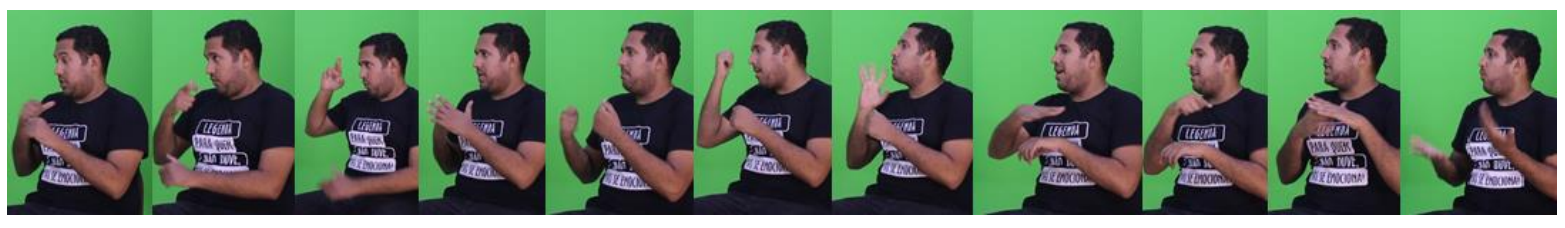

\section{SE EU CHEFE EMPRESA PRECISAR SABER CONHECER [ÁREA IX-3}

EMPRESA QUAL] ${ }^{12}$

'Se eu fosse chefe de empresa, precisaria saber e ter conhecimento sobre qual fosse a área (dela) da empresa.'

\section{[ALGUNS SURDO.SG CRIANÇA.SG-a IX-a] (,) [FAMÍLIA-a IX.PL-a] SABE ESCREVER PORTUGUÊS BOM}

“Algumas crianças surdas, as famílias (delas) escrevem bem em Português"

(Dados primários- vídeo internet)

Sumarizando, podemos ter a seguinte interação entre o contexto e a ordem das apontações:

Tabela 2 - Funções das apontações: ordem de IX vs contexto discursivo

\begin{tabular}{|c|c|c|c|}
\hline & & \multicolumn{2}{|c|}{ FUNÇÕES } \\
\hline & & Primeira-menção & Contextos anafóricos \\
\hline \multirow{3}{*}{ 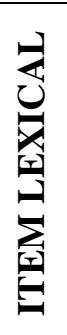 } & $\begin{array}{l}\text { IX-3 art pré- } \\
\text { nominal }\end{array}$ & 1.Artigo definido & $\begin{array}{l}\text { 1.Artigo definido } \\
\text { 2.Pronome possessivo }\end{array}$ \\
\hline & $\begin{array}{l}\text { IX-3 dem pós- } \\
\text { nominal }\end{array}$ & $\begin{array}{l}\text { 1.Demonstrativo } \\
\text { gramaticalizado }\end{array}$ & $\begin{array}{l}\text { 1.Demonstrativo gramaticalizado } \\
\text { 2.Pronome possessivo }\end{array}$ \\
\hline & $\begin{array}{l}\text { IX-3 pro pré e } \\
\text { pós-verbal }\end{array}$ & 1.Pronome pessoal & 1.Pronome pessoal \\
\hline
\end{tabular}

Fonte: elaboração própria (2021).

${ }^{11}$ O bridging é um tipo de "anáfora associativa" (CLARK, 1975), pela qual podemos acessar indiretamente um referente através de outro.

${ }^{12}$ Neste exemplo, cada frame contendo um sinal corresponde exatamente a uma palavra na transcrição. 
Como na nossa proposta, a apontação com função de pronomes pessoais ocorre desacompanhada de nomes, ela não está sujeita à leitura ambígua dos demais tipos de apontação. A ambiguidade só recairia então, para os contextos anafóricos em que se tem um IX com função de artigo ou de demonstrativo gramaticalizado em libras.

Esta problematização também aponta para pesquisas futuras em que se deve analisar duas questões: $i$. qual o limite prosódico, geralmente marcado pelas expressões faciais, que indicam que temos um sintagma nominal, do tipo, 'IX-1 VOVO' que pode significar 'minha vó' e a leitura predicativa em que deve figura alguma quebra prosódica do tipo 'IX-1 tópico, VOVO' que poderia significar 'eu sou avó'; e ii. corolário desta primeira observação é também a necessidade de se investigar em que casos destes temos o redobro do sujeito, do tipo 'IX3tópico, VOVO' que se lê como o 'ela, a vovó' e não com as interpretações de artigo definido ou pronome possessivo como em (8) acima.

\section{A Coordenação de sintagmas e a apontação}

Nesta seção trago outra problematização para a categorização das apontações em libras, considerando, agora, o fenômeno sintático da coordenação em LSs.

Com exceção de Silva (2019) ${ }^{13}$, desconheço descrições na literatura sobre como a libras realiza sintagmas coordenados. O fenômeno da coordenação é relevante para a problematização que faço aqui uma vez que tanto nomes nus ${ }^{14}$, como nomes precedidos da apontação podem ser coordenados em libras. Além disso, o fenômeno da coordenação explora o contraste entre os espaços laterais ao lado do sinalizador, os mesmos que estamos atribuindo à leitura definida dos nomes.

Zorzi (2018a;2018b) explica que a principal maneira como a coordenação é expressa em língua de sinais catalã, LSC, é assindeticamente, por isso, na ausência de uma conjunção, o uso de expressões faciais, EFs, entram em cena para demarcar os limites entre as unidades sintáticas que estão sendo coordenadas. Entre as EFs utilizadas estão a 'inclinação da cabeça' e a 'mudança na posição do tronco para os espaços laterais'. Zorzi afirma que o contraste entre os espaços ipsi- e contralaterais é fundamental para se estabelecer a coordenação. Ou seja, não é possível coordenar duas estruturas sintáticas em LS sinalizando ambos conjuntos no espaço em frente o corpo do sinalizador. Como vemos no exemplo da autora em (11) a seguir, a primeira sentença deve ser sinalizada no espaço contralateral, em contraste como a segunda

\footnotetext{
13 A Autora encontra sinais, possivelmente conjunções, realizadas nos contextos aditivos e adversativos, e a realização de uma pausa entre as orações justapostas. No entanto, os resultados não exploram o uso polarizado do espaço para se codificar a coordenação.

${ }^{14}$ Desacompanhados da apontação pré-nominal de artigo ou de outros determinantes.
} 
sentença, que deve ser sinalizada no espaço ipsilateral. Observe que o espraiamento das EFs se estendem dentro dos limites das unidades sintáticas, como vemos nas linhas sobrescritas indicando os traços suprassegmentais.

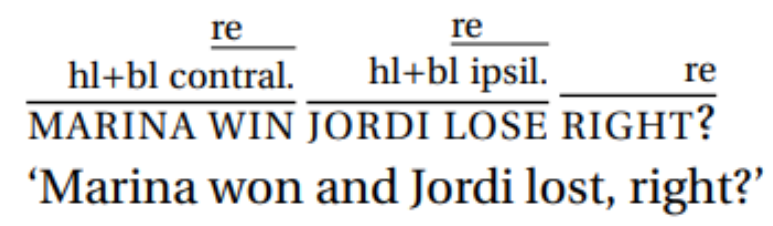

'A Marina venceu e o Jordi perdeu, não foi?'

LSC (ZORZI, 2018a, p. 135)

Tendo em vista que a coordenação envolve o posicionamento dos referentes (no caso de nomes) ou sentenças, nas regiões laterais opostas, o uso de apontações junto aos nomes causa a sensação de que sempre teremos sempre a leitura demonstrativa, e nunca a leitura definida como propus. Mostrarei a seguir que isso não se sustenta para a análise que faço da libras.

Em (12) temos um caso de coordenação de dois nomes na libras, e em (13), um caso em que mais de dois nomes em posição de objeto são coordenados.

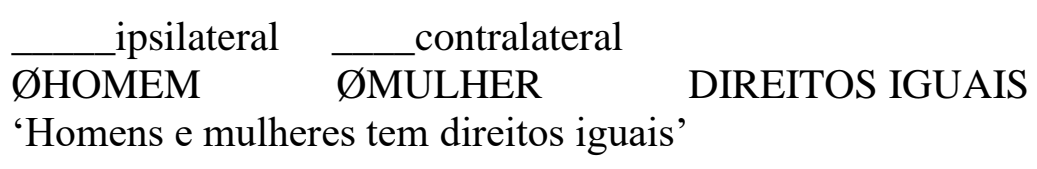

EU IR FEIRA COMPRAR Ø BANANA $\overline{\emptyset M A C ̧ A ̃ ~} \overline{\emptyset T O M A T E}$

'Eu fui à feira e comprei banana, maçã e tomate.

No geral, os sinalizantes não aceitam nomes nus serem realizados numa mesma área no espaço. Se isso ocorre, o sinalizante pode (em algum contexto) presumir que os referentes estavam juntos no mesmo evento, mas nunca serão interpretados como elementos coordenados, isso vale para dois (14) ou mais itens $(15)^{15}$.

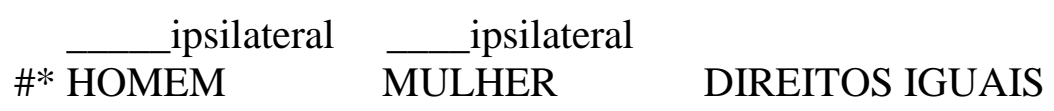

\footnotetext{
${ }^{15}$ Neste caso, como se trata de nomes que são realizados tocando no corpo do sinalizador, observa-se a inclinação do tronco para o lado para que os nomes nus possam ser, assim, localizados lateralmente.
} 
* 'Homens e mulheres tem direitos iguais'

\#? 'Homens e mulheres (que estavam juntos no espaço?) tem direitos iguais'

$$
\begin{aligned}
& \text { \#*EU IR FEIRA COMPRAR BANANA } \frac{\text { ipsilateral }}{\text { MAÇÃ }} \text { ipsilateral } \\
& \text { *'Eu fui à feira e comprei banana, maçã e tomate.' } \\
& \text { \#? 'Eu fui à feira e comprei banana, maçãa e tomate (um kit de frutas?).' }
\end{aligned}
$$

Em libras, os surdos podem também utilizar os sinais de IX para coordenar os nomes no espaço, como em (16). A leitura de IX nestes casos de coordenação parece ser demonstrativa, mas não é, e somente decorreria do fato que se está coordenando (contrastando), no espaço, os elementos que estão posicionados à direita em oposição aos da esquerda. Esta observação se confirma na interpretação das sentenças em (16)-(18), pois a leitura demonstrativa não é licenciada ${ }^{16}$.

ipsilateral

IX-3.PL PROFESSOR (TAMBÉM) IX-3.PL INTÉRPRETE
IGUAIS
'Os/*estes professores e os/*estes intérpretes têm direitos iguais'

\begin{tabular}{l}
\hline IX-3.PL PROFESSOR (TAMBÉM) ${ }^{\circ \text { INTÉRPRETE DIREITO }}$ \\
IGUAIS \\
'Os/*estes professores e os/*estes intérpretes têm direitos iguais'
\end{tabular}

ipsilateral

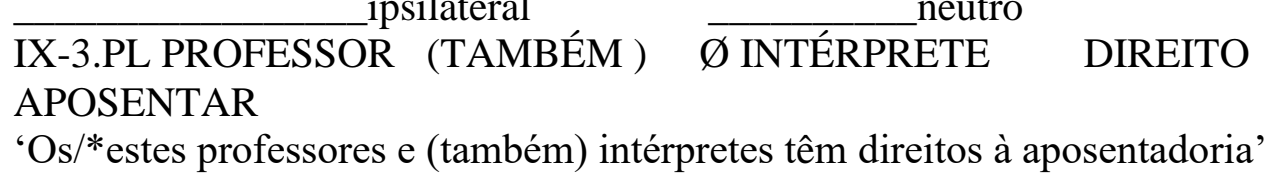

Segundo o Parallel Structure Constraint (restrição das estruturas paralelas) proposto por Lang (1987, tradução minha), numa estrutura de coordenação "os conjuntos coordenados devem ter uma estrutura sintática, semântica e prosódica paralela”, por isso, só se pode coordenar estrutura sintáticas idênticas. Em (17), então, a possibilidade de se coordenar um nome precedido de IX com um nome nu posicionado contralateralmente evidencia que se tratam de dois nomes encabeçados por determinantes, no entanto, o segundo conjunto, por hipótese,

\footnotetext{
${ }^{16}$ Conforme intuição dos surdos entrevistados para esta coleta de dados.
} 
possui um determinante nulo. Contudo, se o segundo nome não for localizado contralateralmente e for realizado no espaço neutro (18), como não há mais coordenação de dois DPs, a leitura agora é a de que 'ser intérprete' é uma propriedade adicional do grupo de professores, esta é a mesma leitura que se tem no PB para sentenças como "A juíza e professora entrou na sala" versus "A juíza e a professora entraram na sala". Novamente, em nenhum destes casos a leitura demonstrativa é permitida.

Verificamos também que nos casos em que quaisquer que sejam as categorias da IX do primeiro elemento coordenado, no caso de elipse nominal (gapping) do segundo elemento, a interpretação é a mesma do elemento no primeiro conjunto ${ }^{17}$, como em (19) e (20).

IX-3.PL PROFESSOR LIBRAS

ipsilateral

(TAMBÉM) IX-3.SG [ ] MATEMÁTICA

contralateral

FALTAR HOJE.

'Os/*estes professores de libras e o/*este prefesseres de matemática faltaram hoje'

$\overline{\text { CASA IX-3.PL }}$ FORTE [ ] $\overbrace{\text { IX-3.PL }}^{\text {contralateral }}$ FRACO

'Estas/*as casas são fortes, estas/aquelas/*as são fracas'

A mesma variação na função de IX dependendo do contexto explicitada na seção anterior A função possesiva da apontação lateral se aplica aos contextos de coordenação de nomes. Nos casos nos quais IX estiver sendo utilizado para retomar referentes já mencionados e que estão sendo contrastados (21), ele pode receber a leitura de pronome demonstrativo, mas isso é variável para os contextos em que os referentes retomados não tenham sido anteriormente apresentados como ALUN@ em (22), ou para os quais não haja pistas suficientes para saber a categoria da apontação, como no exemplo em (23).

${ }_{\text {GRUPO PROFESSOR }}^{\text {ipsilateral }}$

ipsilateral

IX-3.PL SABER LIBRAS contralateral

GRUPO PROFESSOR

contralateral

'Dois grupos de professores, \{eles/estes $\}$ sabem libras, já \{eles/estes\} não sabem'

\footnotetext{
${ }^{17}$ Este exemplo foi amplamente debatido com nossos participantes, pois os mesmos afirmam que sentenças deste tipo não são comuns em proferimentos naturalísticos, mas que essa construção é possível em libras.
} 
(22)

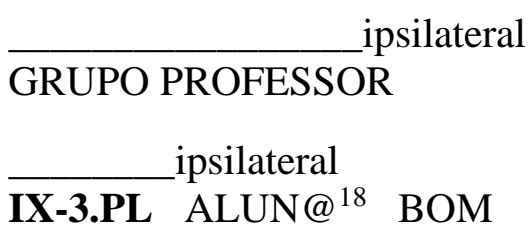

\author{
contralateral \\ GRUPO PROFESSOR \\ contralateral
}

IX-3.PL ALUN@ RUIM

'Dois grupos de professores, $\{$ os/estes $\}$ alunos destes são bons, $\{$ os/estes $\}$ alunos destes são ruins'

ipsilateral

\section{IX-1 GOSTAR IX-3.PL}

\section{contralateral \\ GRUPO PROFESSOR}

contralateral

'Há dois grupos de professores, eu gosto \{deles/destes\} e não \{deles/destes\}'

Este último caso se assemelha ao exemplo de Koulidobrova \& Lillo-Martin (2016), no qual as autoras argumentam sobre a falta de categorização e ambiguidade de IX, que em (24) poderia ser interpretado como um demonstrativo ou um locativo. No entanto, como foi atribuído um locus no espaço ao sinal para RESTAURANTE, como indicado pela glosa de (a-IX), certamente a retomada correferencial por a-IX no final da sentença indicaria que o falante deseja comer 'lá' no restaurante, já que o item COMIDA não se encontra localizado no espaço, pois se estivesse poderia ser retomado com uma apontação e receberia a interpretação de que o falante deseja comer 'isso'. Neste caso, essa apontação seria entendida como uma apontação demonstrativa não-gramaticalizada, aquela em que a interpretação depende do tipo de objeto para o qual está se apontando no espaço real ou mental.

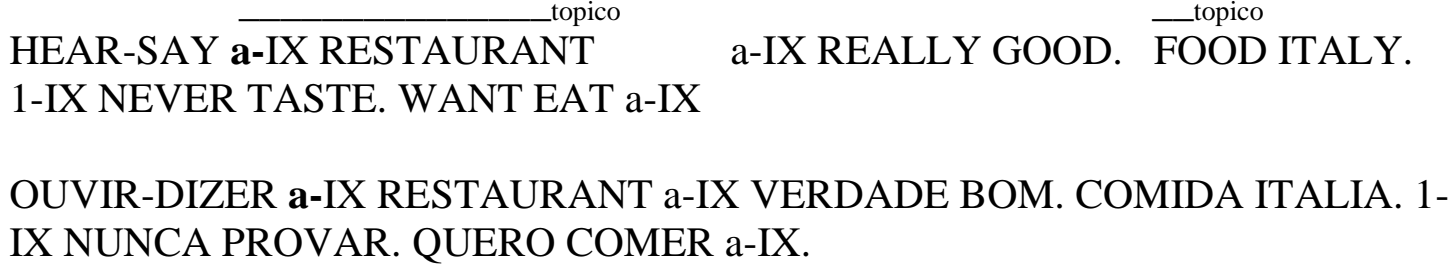

'Eu ouvi dizer que aquele restaurante alí é muito bom. A comida é italiana. Eu nunca provei. Eu quero comer \{aquilo/lá\}.

$$
\text { Koulidobrova \& Lillo-Martin (2016.p244, tradução minha). }
$$

\footnotetext{
${ }^{18} \mathrm{Na}$ transcrição, o @ marca a ausência de gênero do sinal.
} 


\section{Conclusão}

Este texto propôs-se a discutir contextos sintáticos e discursivos nos quais observa-se que a apontação em libras pode variar na leitura categorial dos itens de apontação. Assumindo a proposta de categorização paras as apontações em libras de Almeida-Silva $(2019 ; 2021)$ a exposição problematiza e dialoga com a teoria disponível sobre o fenômeno para analisar se é possível manter a categorização proposta anteriormente pelo autor.

Aqui vimos que a categoria de IX é influenciada não somente pela distinção fonológica e sintática proposta pelo autor, mas pelo contexto em que a apontação ocorre (sentenças-raízes, contextos anafóricos, estruturas de coordenação, entre outros) e ainda pode ter a leitura influenciada pelos traços semânticos do sinal apontado. Contudo, a despeito da aparente variação na leitura categorial dos sinais, a proposta de Almeida-Silva (2019;2021) pode ser mantida.

Com esta problematização, alerta-se para que futuras análises, antes de assumirem categorias morfológicas para os sinais de apontação, percebam, a priori, os contextos sintáticos e discursivos em que o sinal de IX ocorre.

Após as análises, pode-se afirmar que a apontação em LSs é um fenômeno de extrema sensibilidade aos contextos sintáticos e discursivos em que ocorrem, como também de complexidade, tendo em vista uma tendência em se assumir única e inadvertidamente o uso gestual destes.

\section{Referências}

ABNER, N. R. There once was a verb: The predicative core of possessive and nominalization structures in American Sign Language. Los Angeles: University of California, 2012.

ALIBAŠIĆ, T.; WILBUR, R. B. Pronominal system in Croatian sign language. Sign Language \& Linguistics, v. 9, n. 1-2, p. 95-132, 2006.

ALMEIDA-SILVA, Anderson. A (in) definitude no sintagma nominal em libras: uma investigação na interface sintaxe-semântica. 2019. 351 f. Tese (Doutorado em Linguística) Programa de Pós-Graduação em Linguística, Universidade Estadual de Campinas, Campinas, 2019.

ALMEIDA-SILVA, Anderson. Uma proposta de categorização das apontações laterais libras. Cadernos de Linguística, v. 2, n. 4, p. e465, 2021. DOI: https://doi.org/10.25189/26754916.2021.v2.n4.id465.

CHOMSKY, N. Knowledge of language: Its nature, origin, and use. Westport, Connecticut (EUA): Greenwood Publishing Group, 1986. 
CHOMSKY, Noam. The minimalist program. Cambridge (MA): MIT Press, 1995.

CLARK, H. H. Bridging. In: NASH-WEBBER, B. L. PROCEEDINGS OF THE 1975

WORKSHOP ON THEORETICAL ISSUES IN NATURAL LANGUAGE PROCESSING, 1975, Massachusetts. Anais [...]. Massachusetts: Association for Computational Linguistics, 1975. p. 169-174.

CORMIER, K.; FENLON, J. Possession in the visual-gestural modality: How possession is expressed in British Sign Language. In: MCGREGOR, Willian B. (Ed.). The expression of possession. De Gruyter Mouton, 2010. p. 389-422.

KOULIDOBROVA, E.; LILLO-MARTIN, D. A 'point' of inquiry: The case of the (non-) pronominal. In: TRUTKOWSKI, E. et al. The impact of pronominal form on interpretation. Boston/Berlim: De Gruyter Mouton, 2016. p. 221-250.

LANG, E. Parallelismus als universelles Prinzip der Strukturbil dung. In: LANG, E.; SAUER, G. (Eds.). Parallelismus und Etymologie. Berlin: Akademie der Wissenschaften der DDR, Zentralinstitut für Sprachwissenschaft, 1987. p. 1-54.

LIDDELL, S. K. et al. Grammar, gesture, and meaning in American Sign Language. Cambridge: Cambridge University Press, 2003.

SILVA, C. C. Coordenação aditiva e adversativa em libras. 2019. XX f. Dissertação (Mestrado em Linguística) - Programa de Pós-Graduação em Linguística, Universidade de Brasília, Brasília, 2019.

ZORZI, G. Coordination and gapping in Catalan Sign Language (LSC). 2018a. 410 f. Tese (Doutorado em Linguística e Línguas) - Programa de Pós-Graduação em Tradução e Ciências da Linguagem, Universitat Pompeu Fabra, Barcelona, 2018.

ZORZI, G. Coordination in Catalan Sign Language: a syntactic account for conjunction. FEAST, n. 2, p. 132-142, 2018 b.

\section{Sobre o autor}

Anderson Almeida-Silva (Orcid iD: https://orcid.org/0000-0003-4369-4885)

Doutor em Linguística pela Universidade Estadual de Campinas (Unicamp); mestre em Letras - Estudos de Linguagem pela Universidade Federal do Piauí (UFPI); especialista em Libras pelo Instituto Federal do Piauí (IFPI). É professor na Universidade Federal do Delta do Parnaíba (UFDPar).

Recebido em julho de 2021

Aprovado em novembro de 2021. 
ANEXO - DPs acompanhados ou não de apontação encontrados em dados naturalísticos/espontâneos

Legenda: (N) Posição não-argumental; (S) Sujeito; (O) Objeto.

\section{Autores:}

1. Alexandre Melendez

2. Thiago Albuquerque

3. Desconhecido/Sem autorização para divulgação da imagem

4. Desconhecido/Sem autorização para divulgação da imagem

5. Rafael Emil

6. Desconhecido/Sem autorização para divulgação da imagem

7. Desconhecido/Sem autorização para divulgação da imagem

8. Antônio Campos

9. Williane Souza

10. Thiago Albuquerque

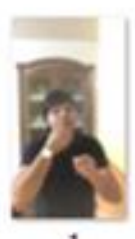

1

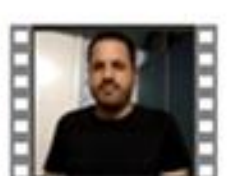

2

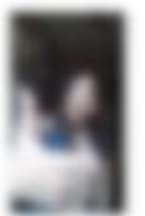

4

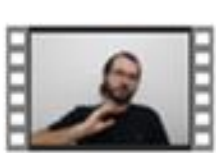

5

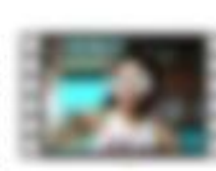

6

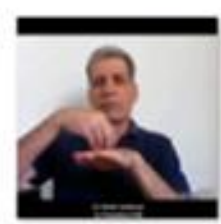

8

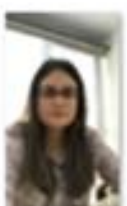

9

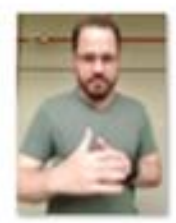

10 
Revista (Con)Textos Linguísticos, Vitória, v. 15, n. 32, p. 201-221, 2021 | e-ISSN 1982-291X | ISSN 2317-3475 Revista do Programa de Pós-Graduação em Linguística da UFES | periodicos.ufes.br/contextoslinguisticos

\begin{tabular}{|c|c|c|c|c|c|c|c|}
\hline Vídeo/Minutos & 1 ' & $2^{\prime}$ & $3^{\prime}$ & $4^{\prime}$ & $5^{\prime}$ & $6^{\prime}$ & $7^{\prime}$ \\
\hline Vídeo 1 & 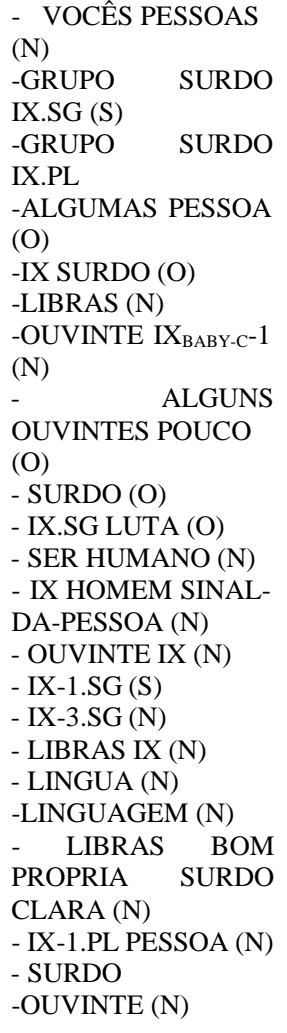 & $\begin{array}{l}\text { - QUALQUER PESSOA } \\
\text { (S) } \\
\text { - CONSCIÊNCIA (O) } \\
\text { - IX-1.SG (S) } \\
\text {-LETRAS-LIBRAS (O) } \\
\text {-IX-1.SG (S) } \\
\text { - PORTUGUÊS (O) } \\
\text {-ALGUNS SURDOS } \\
\text { CRIANÇA IX (N) } \\
\text {-FAMILIA IX.PL (S) } \\
\text { - PORTUGUÊS (O) } \\
\text { - L2 (N) } \\
\text { - PROFISSIONAL ÉTICA } \\
\text { (N) } \\
\text {-RESPONSABILIDADE } \\
\text { MEU (O) } \\
\text {-IX.PL SURDO (O) } \\
\text { - IX-1.SG (S) } \\
\text {-IX.SG OUVINTE } \\
\text { - IX.PL ESCOLA } \\
\text { DENTRO OUVINTE } \\
\text { IX.SG (O) OUTU (O) } \\
\text { - PORTUGUÊS } \\
\text { - IX.SG OUVINTE (N) } \\
\text { - L2 LIBRAS (N) } \\
\text { - SURDO (S) } \\
\text { - SURDO (S) } \\
\text { - PORTUGUÊS (O) }\end{array}$ & $\begin{array}{l}- \\
\text { DIFERENÇA CADA } \\
\text { LÍNGUA (O) } \\
-\quad \text { QUALQUER } \\
\text { PESSOA (N) } \\
\text { - L2 FRANCES (N) } \\
\text {-L1 PORTUGUES } \\
\text { (N) } \\
\text {-L1 LIBRAS (N) } \\
\text { - L1 INGLES (N) } \\
\text { - TODOS (S) } \\
\text { - IX-3.SG (N) } \\
\text { - INGLÊS (N) } \\
\text { - IX SURDO (S) } \\
\text { - IX-1 (S) } \\
\text { - MAIORIA } \\
\text { LIBRAS SURDO(O) } \\
\text { - EXEMPLO (N) } \\
\text { - TEMA (N) } \\
\text { - ENEM (N) } \\
\text { - IX TEMA (S) } \\
\text { - LIBRAS L2 (O) } \\
\text { - L1 IX.SG (N) } \\
\text { - PORTUGUÊS (N) } \\
\text { - L2 (O) } \\
\text { - ENEM LIBRAS } \\
\text { (O) } \\
\text { - IX ENEM (S) } \\
\text { - SURDO IX (O) } \\
\text { - MAIORIA SURDO } \\
\text { IX LIBRAS (O) } \\
\text { - LIBRAS IX (N) }\end{array}$ & $\begin{array}{l}\text {-OUVINTE(S) } \\
\text { - L2 DELE (O) } \\
\text { - IX-1 (S) } \\
\text { - PROLIBRAS IX (S) } \\
\text { - IX.PL OUVINTE (S) } \\
\text { - LIBRAS FRACA (N) } \\
\text { - IX PESSOA SURDA (O) } \\
\text { - OUVINTE (O) } \\
\text { - CONSCIÊNCIA (O) } \\
\text { - IX HOMEM IX } \mathrm{O} \text { BABY-C-3 } \\
\text { IX (S) } \\
\text { - IX } \text { BABY-C-3 (O) }_{\text {- }} \\
\text { - PROBLEMA (N) } \\
\text { - IX-1.PL } \\
\text { - CONHECIMENTO (O) } \\
\text { - MATURIDADE (N) } \\
\text { - INFORMAÇÃO (N) } \\
\text { - EDUCAÇÃO SURDO } \\
\text { IX (O) } \\
\text { - BILINGUISMO (O) } \\
\text { - IDENTIDADE SURDA } \\
\text { (O) } \\
\text { - LIBRAS }\end{array}$ & $\begin{array}{l}\text { - OUVINTES (S) } \\
\text { - IX LIBRAS (N) } \\
\text { - DISCUSSÃO IX (N) } \\
\text { - INFORMAÇÃO (O) } \\
- \text { SENTIMENTO IX } \\
\text { POESIA (O) } \\
-\quad \text { TRADUÇÃO } \\
\text { QUALQUER (N) } \\
\text { - IX LINGUA (O) } \\
\text { - IX PORTGUES (N) } \\
\text { - LIBRAS IX } \\
-\quad \text { IX.PL SURDOP } \\
\text { GRUPO (O) }\end{array}$ & & \\
\hline Vídeo 2 & $\begin{array}{l}\text { - DEUS (N) } \\
\text { - BÍBLIA (N) } \\
\text {-IGREJA(N) } \\
\text {-IX-1.SG(S) } \\
\text { - TEMPO (O) } \\
\text {-TRABLAHO(O) } \\
\text {-ESTUDO (O) } \\
\text {-IX-2.PL (S) } \\
\text {-TEMPO(O) } \\
\end{array}$ & $\begin{array}{l}\text { - IX PESSOA GERAL } \\
\text { OPINÃO PERGUNTA(N) } \\
\text { - PERGUNTA (N) } \\
\text {-PESSOA (O) } \\
\text {-IX.1.SG (S) } \\
\text {-DEUS(O) } \\
\text {-IGREJA (O) } \\
\text { - BÍBLIA (N) }\end{array}$ & $\begin{array}{l}\text {-IX.SG (S) } \\
\text { - DEUS (O) } \\
\text { - IX-2.SG (S) } \\
\text { - IX.SG DEUS (O) } \\
\text { - DEUS (O) } \\
\text {-COISAS (O) } \\
\text {-DEUS (OI) } \\
\text { - HISTÓRIA DELE } \\
(\mathrm{O}) \\
\end{array}$ & $\begin{array}{l}\text { - SABEDORIA (N) } \\
\text { - INTELIGÊNCIA (N) } \\
\text { - IX BÍBLIA (N) } \\
\text { - VIDA PASSADO (N) } \\
\text { - DIA (N) } \\
\text { - TRABALHO BOM }(\mathrm{O}) \\
\text { - FAMÍLIA BOM }(\mathrm{O}) \\
\text { - CASAMENTO BOM } \\
(\mathrm{O}) \\
\end{array}$ & $\begin{array}{l}\text {-IX BÍBLIA (S) } \\
\text { - VIDA (O) } \\
-\quad \quad \quad \text { ALGUMAS } \\
\text { PESSOAS (O) } \\
\text { - IGREJA (O) } \\
\text { - IX-2.SG (S) } \\
\text { - IX-3.SG (O) } \\
\text { - IX.SG IGREJA (O) }\end{array}$ & $\begin{array}{l}\text {-ORIENTAÇÃO (O) } \\
\text { - ENSINO (O) } \\
\text { - VIDA (N) } \\
\text {-LOUVOR (N) } \\
-\quad \text { QUALQUER } \\
\text { LUGAR (N) } \\
\text { - PESSOAS INVEJA } \\
\text { (O) } \\
\text { - IGREJA (O) }\end{array}$ & $\begin{array}{l}\text { - QUEM (S) } \\
\text { - IX-1.SG (O) } \\
-\quad \quad \text { PESSOAS } \\
\text { ALGUMAS (O) } \\
\text { - IX-3.PL (S) } \\
\text { - IGREJA (S) } \\
\text { - FELICIDADE (O) } \\
\text { - QUUALQUER } \\
\text { LUGAR (N) } \\
\end{array}$ \\
\hline
\end{tabular}




\begin{tabular}{|c|c|c|c|c|c|c|c|}
\hline & -PESSOA (O) & $\begin{array}{l}\text { - ORAÇÃO (N) } \\
\text { - DEUS (O) } \\
\text { - IX.2.SG (S) } \\
\text { - DEUS (O) } \\
\text { - CÉU (O) }\end{array}$ & $\begin{array}{l}\text { - MÃE MINHA (O) } \\
\text {-PAI MEU (O) } \\
\text { - IX-2.SG (S) } \\
\text {-DEUS(N) } \\
\text { - PESSOAS AÍ (O) } \\
\text { - BÍBLIA IX (N) } \\
\text { - IX-2.SG MESMO } \\
\text { IX }_{\text {BABY---2 (S) }} \\
\text { - BÍBLIA (O) } \\
\text { - PESSOA IX }{ }_{\text {BABAY- }} \\
\text { c++ (S) } \\
\text { - BÍBLIA (O) } \\
\text { - FIM MUNDO (O) } \\
\text { - } \quad \text { COISAS } \\
\text { ABSURDAS (N) } \\
\text { - BÍBLIA (O) } \\
\text { - IX } \\
\text { - IXABY-C++ (O) } \\
\text { - IX BÍBC-CIA (S) } \\
\text { - IX-2.SG (S) } \\
\text { - BÍBLIA (O) } \\
\text { - IX BÍBLIA (N) } \\
\text { - IX BÍBLIA (S) } \\
\text { - C CAMINHO } \\
\text { CERTO (O) } \\
\text { - DEUS (N) } \\
\text { - JESUS (N) } \\
\text { - HISTÓRIA } \\
\text { COISAS (O) } \\
\text { - IX BÍBLIA (S) }\end{array}$ & $\begin{array}{l}\text { - CASA TUDO (O) } \\
\text { - PAI (S) } \\
\text {-MÃE(S) } \\
\text { - MOTIVO (N) } \\
\text { - MANHÃ (O) } \\
\text { - IGREJA (O) } \\
\text { - BÍBLIA (O) }\end{array}$ & $\begin{array}{l}\text { - IX } \\
\text { (N) } \\
\text { - BÍBLIA (O) } \\
\text { - VIDA (N) } \\
\text { - CONQUISTA (N) } \\
\text {-SUPERAÇÃO (N) } \\
\text { - HISTÓRIA (N) } \\
\text { - IX.SG IGREJA (S) }\end{array}$ & $\begin{array}{l}- \text { PESSOAS OLHOS } \\
\text { BRILLHAR (O) } \\
-\quad \text { ALGUMAS } \\
\text { PESSOA (O) } \\
\text { - IGREJA (O) } \\
-\end{array}$ & $\begin{array}{l}\text { - BAR (N) } \\
\text { - INVEJA (N) } \\
\text {-COISAS RUINS (N) } \\
\text { - IGREJA (N) } \\
\text { - IX-2.PL (S) } \\
\text {-IGREJA (O) } \\
\text { - DEUS IX.SG (S) } \\
\text { - IX2.SG (O) } \\
\text { - O-QUE (S) } \\
\text { - BÍBLIA (O) } \\
\text { - O-QUE (S) } \\
\text { - IGREJA (O) } \\
\text { - DEUS (N) } \\
\text { - IX-2.PL (O) } \\
\text {-DEUS (S) }\end{array}$ \\
\hline Vídeo 3 & $\begin{array}{l}\text {-SOCIEDADE } \\
\text { LIBRAS (N) } \\
\text { - ALGUNS (S) } \\
\text { - PENIS TAMANHO } \\
\text { (O) } \\
\text { - IX-1.SG (S) } \\
\text { - HISTORIA UM } \\
\text { HOMEM IX IXABY-C-3 } \\
\text { (O) } \\
\text {-HOMEM UM (N) } \\
\text { - MULHER GERAL } \\
\text { (S) } \\
\text { - MULHER UM (S) } \\
\text { - HOMEM (S) } \\
\text { - MOTEL (O) } \\
\text { - MULHER (S) }\end{array}$ & $\begin{array}{l}\text {-MULHER UM (N) } \\
\text { - GRANDE PENIS (O) } \\
\text { - MULHER (S) } \\
\text { - HOMEM (S) } \\
\text { - BATE-PAPO (N) } \\
\text { - HOMEM (S) } \\
\text { - MULHER (S) } \\
\text { - HOMEM (S) } \\
\text { - FILME (O) } \\
\text { - PASSEIO (O) } \\
\text { - MULHER IX-3.SG (S) } \\
\text { - IX-3.SG } \\
\text { - HOMEM (S) } \\
\text { - UM COISA (O) }\end{array}$ & $\begin{array}{l}\text { - CASAMENTO (N) } \\
\text { - FAMÍLIA (N) } \\
\text {-UMA COISA (O) } \\
\text { - IX-2.SG (O) } \\
\text { - HOMEM (S) } \\
\text { - IX-1.SG (S) } \\
\text { - IX-3.SG (O) } \\
\text { - MULHER (N) } \\
\text { - IX-1.SG (S) } \\
\text { - PROBLEMA (O) } \\
\text { - PROBLEMA (N) } \\
\text { - PENIS (N) } \\
\text { - VIDA (O) } \\
\text { - HOMEM (N) } \\
\text { - MULHER (S) }\end{array}$ & $\begin{array}{l}\text { - MULHER IX (N) } \\
\text { - HOMEM (S) } \\
\text { - IX-2.SG (S) } \\
\text { - PENIS GRANDE (N) } \\
\text { - IX-1.SG (S) } \\
\text { - HOMEM (N) } \\
\text {-IX } \text { IABY-C-1 (O) }_{\text {- IX-3.SG (N) }} \\
\text {-PENIS GRANDE (N) } \\
\text { - MÉDICO (O) } \\
\text { - MÉDICO (S) } \\
\text { - IX-3.SG (S) } \\
\text { - IX-2.PL (S) } \\
\text { - FILHO (S) } \\
\text { - HISTÓRIA (N) }\end{array}$ & & & \\
\hline
\end{tabular}




\begin{tabular}{|c|c|c|c|c|c|c|c|}
\hline & $\begin{array}{l}\text { - HOMEM (S) } \\
\text { - MULHER (N) } \\
\text { - HOMEM IX } \\
\text { (O) } \\
\text { - IX-3.SG (S) } \\
\text { - GRANDE PENIS (O) } \\
\text { - MULHER GERAL } \\
\text { (S) } \\
\text { - HOMEM (S) }\end{array}$ & & $\begin{array}{l}- \text { - PENIS GRANDE } \\
(\mathrm{N}) \\
-\quad \text { MULHER } \\
\text { VARIAS (N) } \\
- \text { PENIS GRANDE } \\
(\mathrm{N}) \\
-\quad \text { MULHER } \\
\text { VARIAS (N) } \\
\text { - IX-1.SG (S) } \\
- \text { TODOS (S) } \\
\text {-IX-1.SG (O) } \\
\text {-GRANDE PENIS } \\
\text { (O) } \\
- \text { MULHER (S) }\end{array}$ & $\begin{array}{l}-\quad \text { PENIS GRANDE } \\
\text { PEQUENO (N) } \\
\text { - BOM AJUDAR SAÚDE } \\
\text { (O) } \\
\text { - CARINHO (N) } \\
\text { - AMOR (N) } \\
- \\
\text { COMPARTILHAMENTO } \\
\text { (N) }\end{array}$ & & & \\
\hline Vídeo 4 & $\begin{array}{l}\text {-TODOS SURDOS (N) } \\
\text { - IX-1.SG (S) } \\
\text { - LÁ MULHER (N) } \\
\text { - IX-1.SG (S) } \\
\text { - SINAL (O) } \\
\text { - IX-1.SG (S) } \\
\text { - NOME (O) } \\
\text { - IX.SG IX IXABY-C-3 } \\
\text { MULHER (S) } \\
\text { - DELA DENTRO } \\
\text { HOSPITAL LÁ (O) } \\
\text { - BEBÊ (O) } \\
\text { - IRMÃ IX.SG (N) } \\
\text { - SURDO (N) } \\
\text { - IX-1.SG (S) } \\
\text { - HOSPITAL (O) } \\
\text { - OUTRO LUGAR (N) } \\
\text { - IX-2.SG (S) } \\
\text { - IX-1.SG IX IXABY-C-1 } \\
\text { SURDO (O) } \\
\text { - FAMÍLIA DURA (N) }\end{array}$ & $\begin{array}{l}\text { - IX-1.SG (S) } \\
\text { - COMPUTADOR (O) } \\
\text { - MEU PRIMO (S) } \\
\text { - COMPUTADOR (N) } \\
\text { - COISAS DELE(O) } \\
\text { - PRIMO (S) } \\
\text { - MÃE (O) } \\
\text { - IX-3.SG (S) } \\
\text {-MÂE (S) } \\
\text {-PRIMO (S) } \\
\text { - IX-1.SG IX } \text { BABY-C-1 }_{\text {SURDO (O) }} \\
\text { - IX-2.SG (S) } \\
\text { - MINHA VIDA (O) } \\
\text { - UM OUTRA [N] (N) } \\
\text { - DICIONÁRIO CD (O) } \\
\text { - CD (O) } \\
\text { - PRIMO (S) }\end{array}$ & $\begin{array}{l}\text {-IX-1.SG (S) } \\
\text { - IX-3.SG (S) } \\
\text { - SURDO (O) } \\
\text { - COISAS (O) } \\
\text { - IX-2.SG (S) } \\
\text { - IX.3SG (N) } \\
\text { - FAMÍLIA (S) } \\
\text { - SURDO (O) } \\
\text { - IX-3.PL (S) } \\
\text { - IX } \text { BABAY--1 (O) } \text { - IX-1.SG (S) } \\
\text { - MAMÃE (O) } \\
\text { - IX-1.SG (S) } \\
\text { - LÁ (O) } \\
\text { - AMIGA MULHER } \\
\text { (O) } \quad \text { - IX-3.SG (S) } \\
\text { - MAMÃE (S) } \\
\text { - QUALQUER } \\
\text { LUGAR (N) } \\
\text { - IX-1 IX } \text { BABY-C-1 (O) } \\
\text { - IX-1.SG (S) } \\
\text { - IDENTIDADE } \\
\text { SURDA (O) } \\
\text { - TODOS } \\
\text { MATERIAL (N) }\end{array}$ & 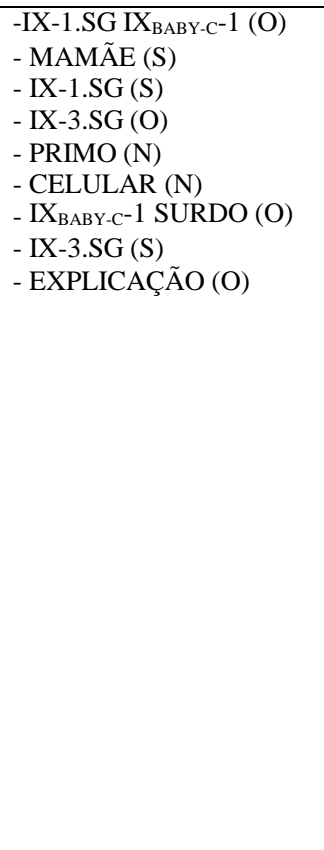 & $\begin{array}{l}\text { - MINHA FAMÍLIA } \\
\text { (S) } \\
\text { - TUDO (N) } \\
\text { - FAMÍLIA (S) } \\
\text { - IX } \text { XABY-C-1 (O) }_{\text {- PROCESSO (N) }} \\
\text { - FAMILIA (N) } \\
\text { - SAÚDE (N) } \\
\text { - MEU NOME (S) }\end{array}$ & & \\
\hline Vídeo 5 & $\begin{array}{l}\text {-TEMA(S) } \\
\text {-PROLIBRAS }(\mathrm{O}) \\
\text { - VÁRIAS PESSOAS } \\
\text { (S) } \\
\text { - PROLIBRAS }(\mathrm{O})\end{array}$ & $\begin{array}{l}\text {-QUANTOS (O) } \\
\text { - } 3 \text { ANOS (N) } \\
\text { - IX-3.SG (N) } \\
\text { - MOTIVO (N) } \\
\text { - IX.3-SG (S) }\end{array}$ & $\begin{array}{l}\text {-CURSOS++ }(\mathrm{O}) \\
\text { - IX CURSO (S) } \\
\text {-TEORIA }(\mathrm{O}) \\
\text { - SINTAXE }(\mathrm{N})\end{array}$ & -PÓS LIBRAS (N) & & & \\
\hline
\end{tabular}




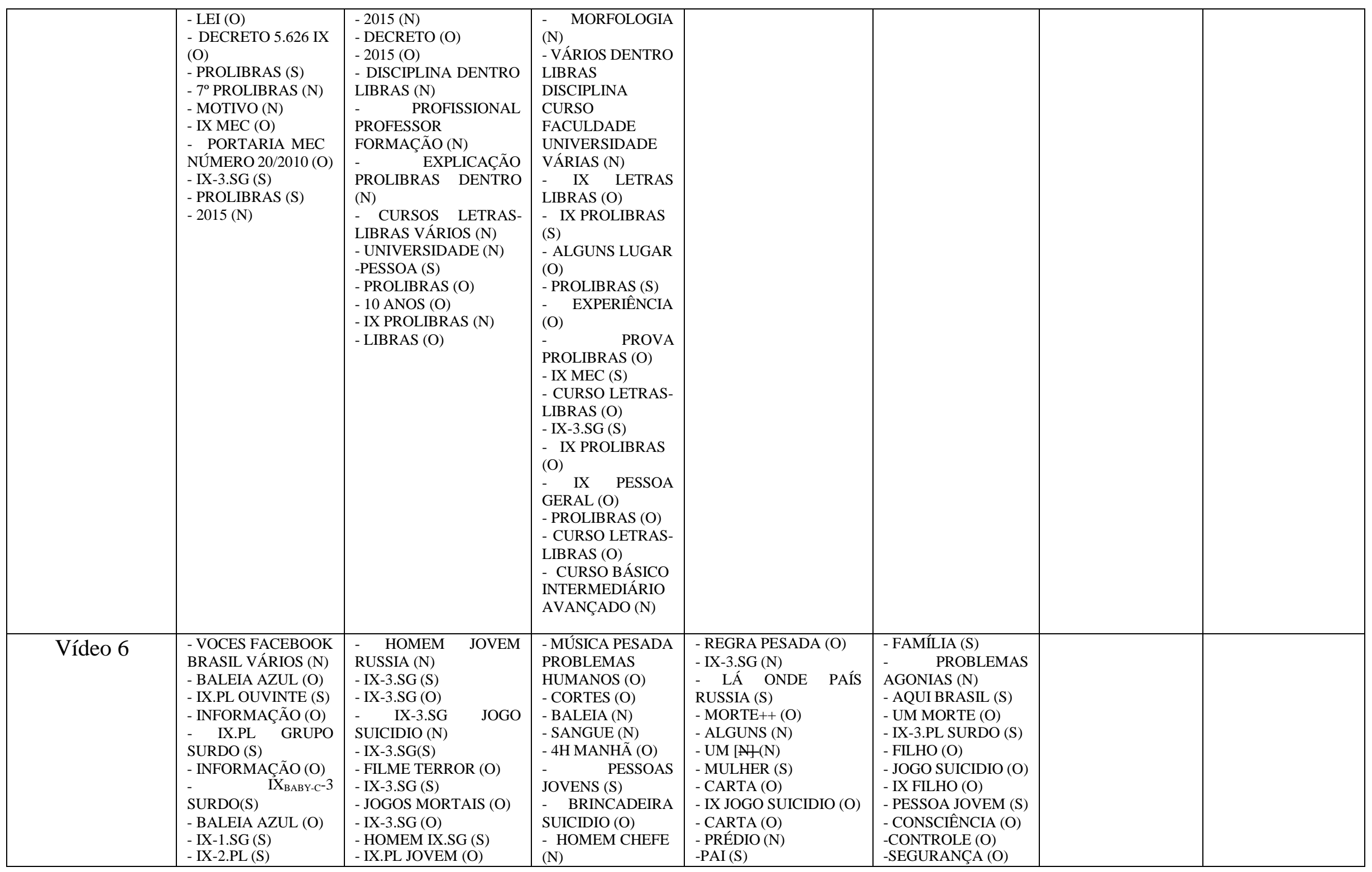




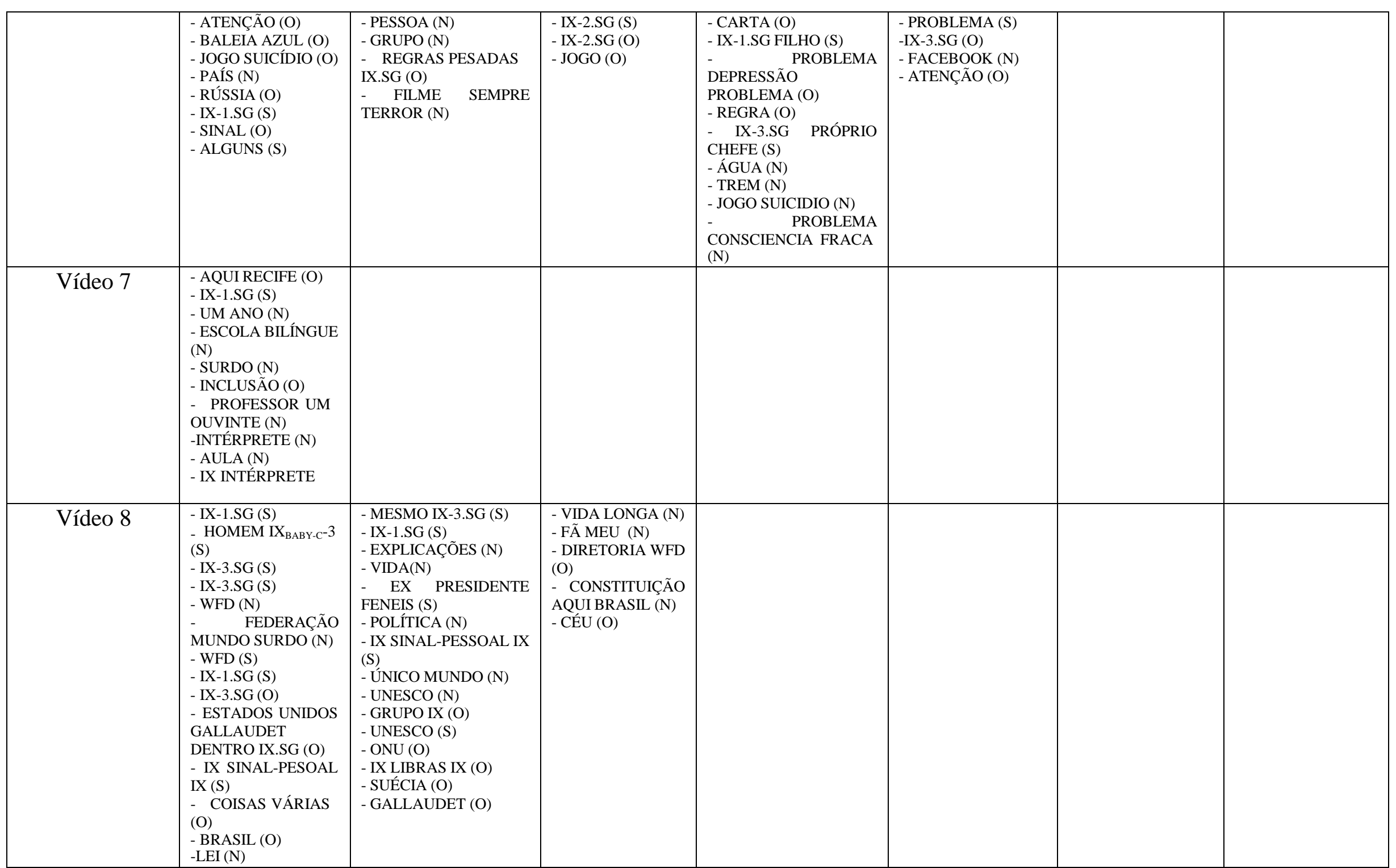


Revista (Con)Textos Linguísticos, Vitória, v. 14, n. 27, p. XX-XX, 2020 | e-ISSN 1982-291X | ISSN 2317-3475

Revista do Programa de Pós-Graduação em Linguística da UFES | periodicos.ufes.br/contextoslinguisticos

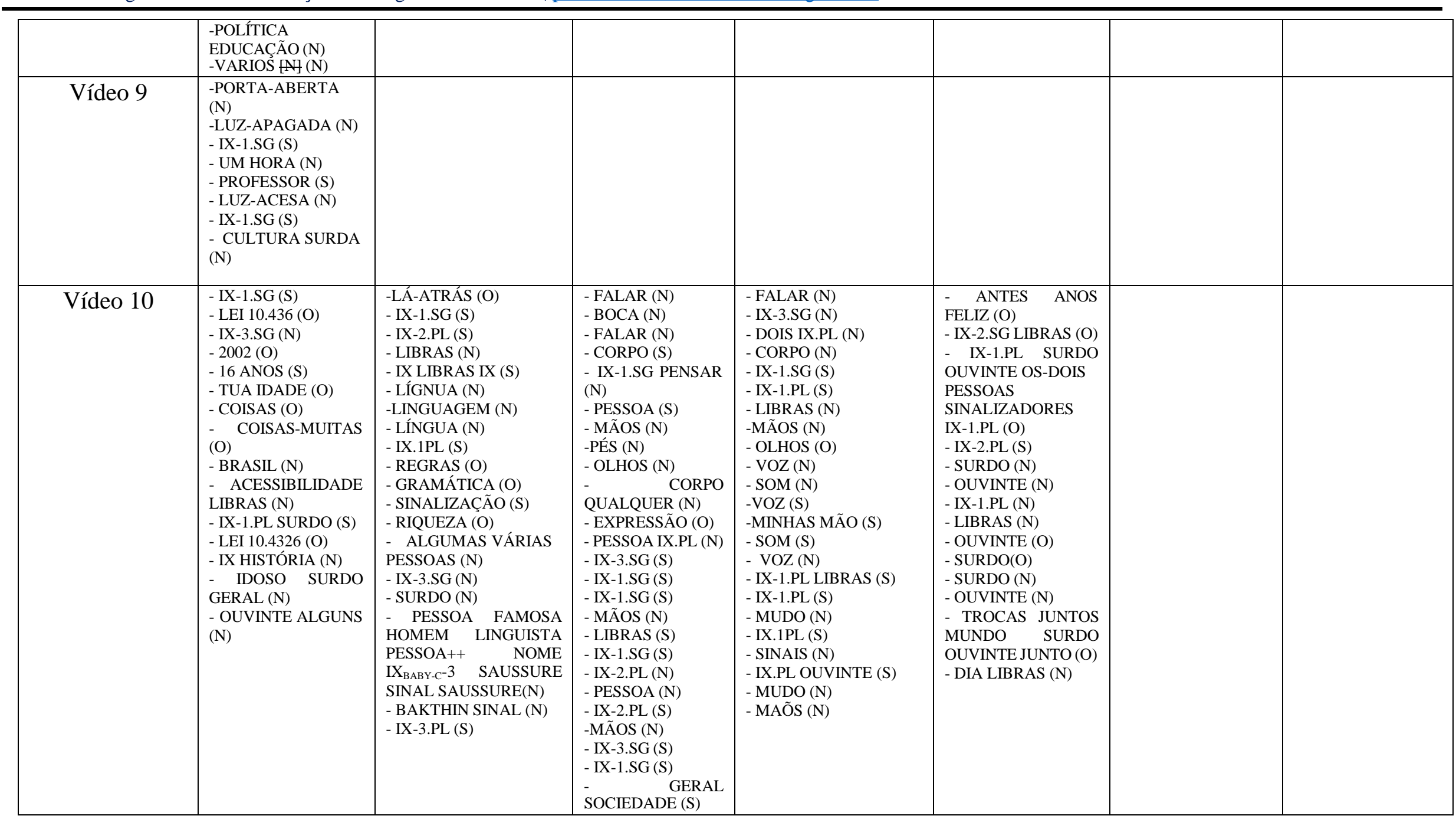

Fonte: Almeida-Silva (2019, p. 190-195). 